www.conferenceie.ase.ro

\title{
BIG DATA INTEGRATION OF SMART GRID CONSUMPTION DATA
}

\author{
Vlad DIACONIT,A \\ Bucharest University of Economic Studies, Romania \\ diaconita.vlad@ie.ase.ro \\ Iuliana ŞIMONCA (BOTHA) \\ Bucharest University of Economic Studies, Romania \\ iuliana.botha@ie.ase.ro \\ Alexandra CORBEA (FLOREA) \\ Bucharest University of Economic Studies, Romania \\ alexandra.florea@ie.ase.ro
}

\begin{abstract}
In the energy field, in the context of the constant and significant growth of smart devices and smart meters usage, there are constant streams of data that should be properly captured, analyzed and taken advantage of.

The article focuses on the presentation of the integration process of a data stream received from smart meters, using Spark, as part of the research conducted within the SMARTRADE project for developing an informatics system prototype for forecasting, analysis and decision models for electricity suppliers in order to estimate electricity consumption and production efficiently.
\end{abstract}

Keywords: Big Data Technologies, Apache Spark, Big Data Analytics, Smart Metering Systems

JEL classification: C88, L88

DOI: $10.12948 / \mathrm{ie} 2019.03 .09$

\section{Introduction}

This paper presents some research conducted within the SMARTRADE (Intelligent System for Trading on the Wholesale Electricity Market) research project, which has at objective design and development of an informatics system prototype for forecasting, analysis and decision models for electricity suppliers in order to estimate consumption and production in an appropriate way for efficient transactions in the wholesale energy market.

As we have detailed in [1], the smart grid concept consists of a combination of advanced equipment and systems used for intelligent metering, as well as consumer devices equipped with sensors, data acquisition systems, automated complex decision support systems, and so on.

This paper focuses on data integration, exemplifying the process of integrating streaming data with static data that is received from smart meters, as shown in Fig. 1.

\section{Big Data overview}

At the wholesale energy market, there are limitations due to the incapacity of current informatics systems used by energy providers and network operators to process smart meter data. At present, the volume of these data is steadily rising, leading to the need for strong platforms and appropriate processing technologies. These data are also characterized by speed, variety and veracity, for which it is necessary to use Big Data technologies and parallel processing with potential applications in intelligent measurement [2]. 
www.conferenceie.ase.ro

The most obvious feature of Big Data is the volume. Increasingly, people use smart devices that are connected to an Internet network and produce data every second. In particular, in the energy field, smart meters continuously record data on consumer energy consumption. Big Data has become a paradigm that involves our entire universe in collecting and processing data. Therefore, it is important to take advantage of these data streams, storing and processing them properly.

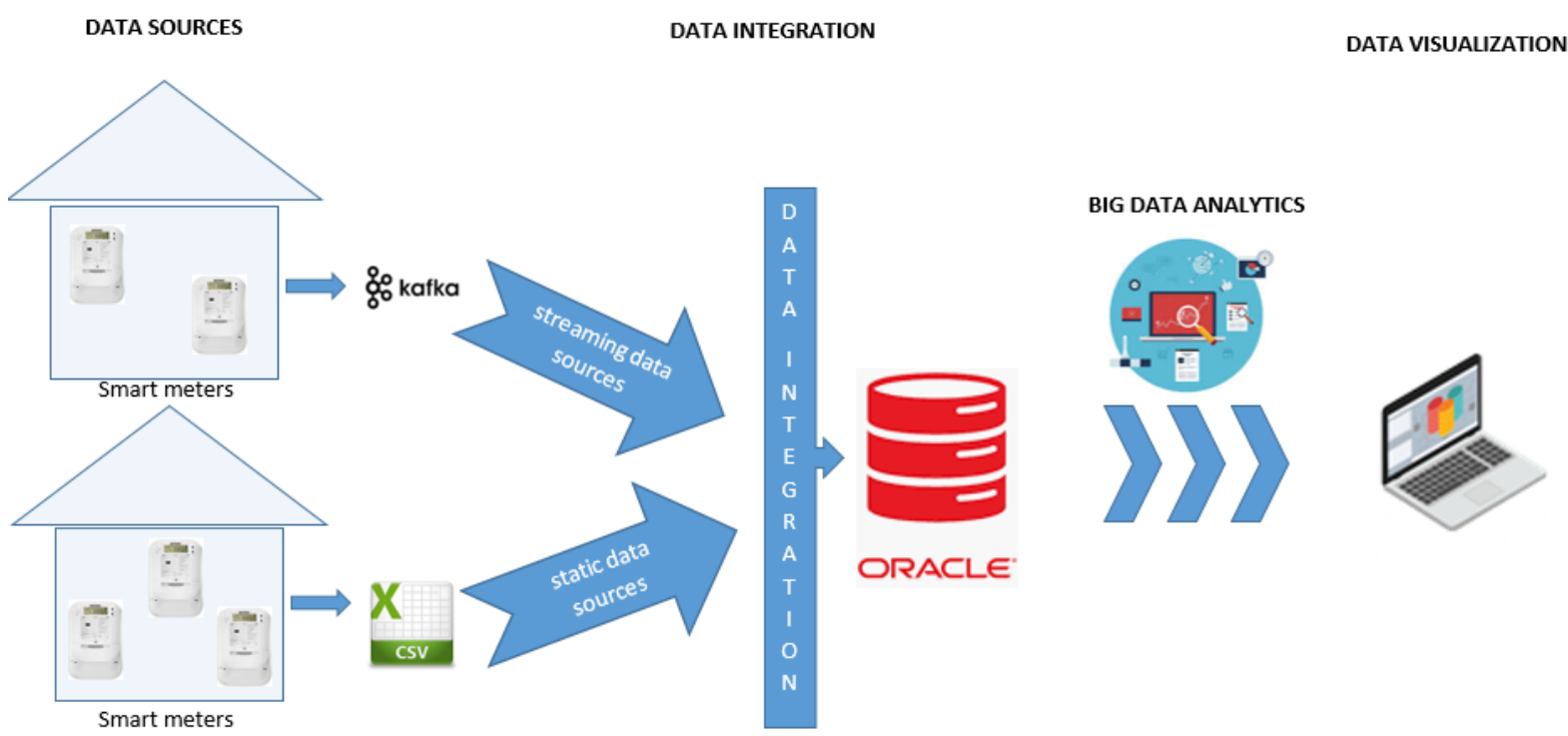

Figure 1. The data flow discussed in the paper

Big Data can be classified from several points of view. So, we're talking about internal or external data coming from the outside of the company, but which are useful to the business model of the firm. From the point of view of data generation, there are deliberately generated data or automatically generated data by various devices: sensors, mobile phones, digital cameras, satellites, etc. From the point of view of the internal data structure [3], we can encounter structured data (that complies with a specific rule or model of internal organization), unstructured data (that does not follow a pre-defined organizational pattern) and semistructured data (that has a poor form of structuring, which it sometimes respect only roughly). From the point of view of dynamics, we can identify static data and streaming data.

For this article the data is flowing from gateways that receive them from smart meters. A smart meter can service different devices or group of devices from within a house: cellar outlets, disposal dishwasher, kitchen light, furnace heat recovery ventilators, refrigerator, etc. The readings are usually received every 1 -minute. The reading contains the timestamp and a value for each device covered by the respective smart meter. Such data can be accompanied by other smart-home specific data like motion sensors, door sensors and even weather-related readings. The granularity of data and the format usually varies. For example, the date of the reading can be expressed in Unix timestamp (seconds that passed from January 1st, 1970 at UTC) or in different formats such as ISO 8601, RFC 822, 1036, 1123, 2822, 3339 or vendor specific formats that can include up to 9 milliseconds (e.g. Oracle Timestamp), e,g.:
- 2016-10-31
19:30:00,
$0.11425,1.6667 e-05,0.00115,1.6667 e-05,1.6667 e-$ $05,0.003483333,0.0047,0.002,0.013883333,1.6667 e-05$
$\begin{array}{lllllll}\bullet & 1341048300,73.580009,59.269997,45.0 & , 83.699997,0.3 & , 105.0 & , 1.0 & , 293.0\end{array}$ 
www.conferenceie.ase.ro

\section{Data Integration Flow}

We used Spark to process and integrate data. Spark has a unified API for different programming languages: Scala, Python, Java and R. It can run on diverse environments ranging from Hadoop to Kubernetes but also as a standalone solution. For fault-tolerance, Spark uses in-memory Resilient Distributed Datasets (RDDs) for the transformations. If a partition is lost, the RDDs can re-run the associated tasks. Spark implements Datasets which are collections of objects on which we can apply transformations and actions.

The data was received as log files in a folder, and in a publish-subscribe pattern on a channel (Kafka). Figure 2 shows how to read data from a file, transform it and load it into a database:

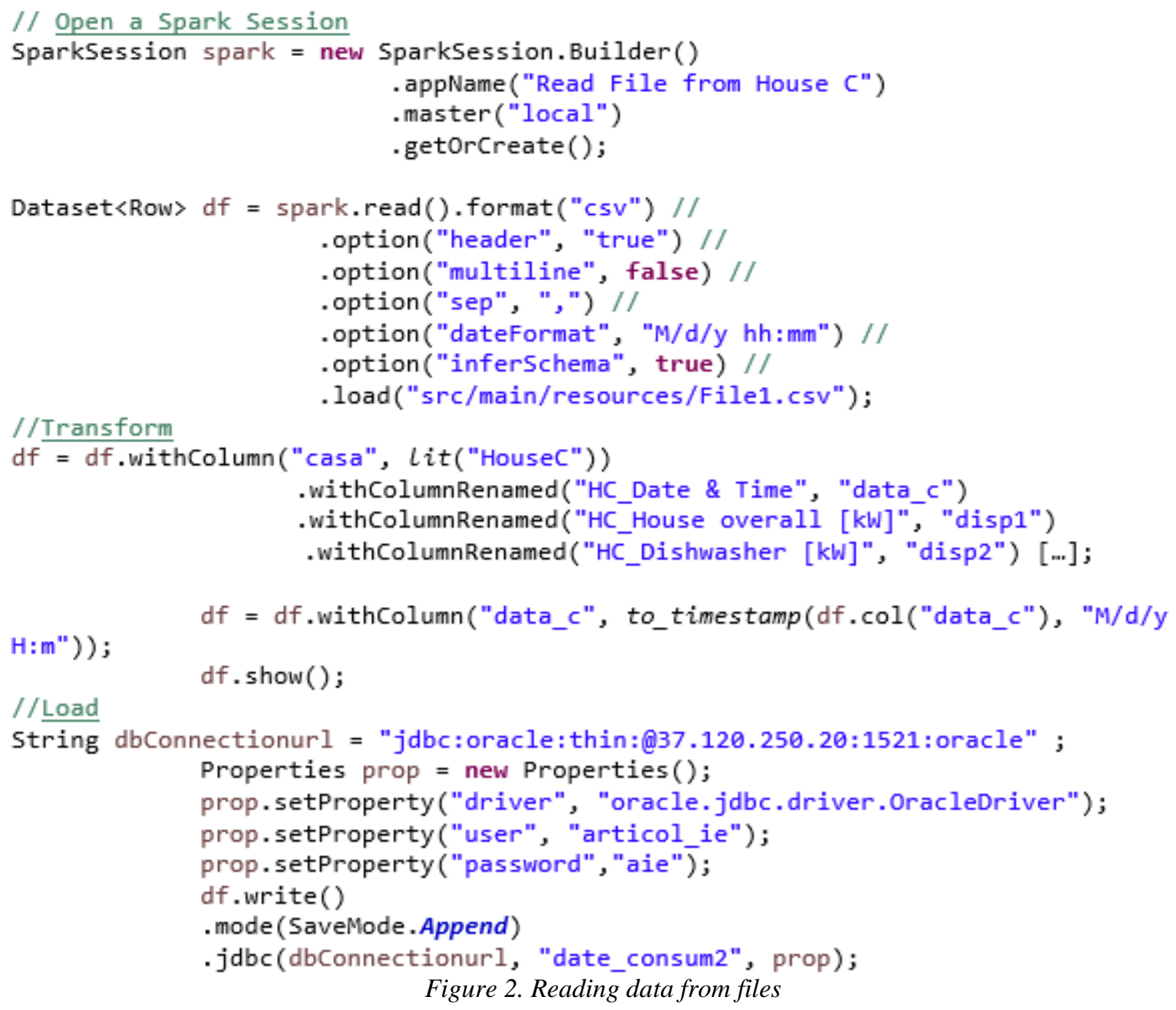

To read data coming from a different gateway on a Kafka channel, the main difference from the previous approach is in how we define the Dataset (as shown in Figure 3): 
www.conferenceie.ase.ro

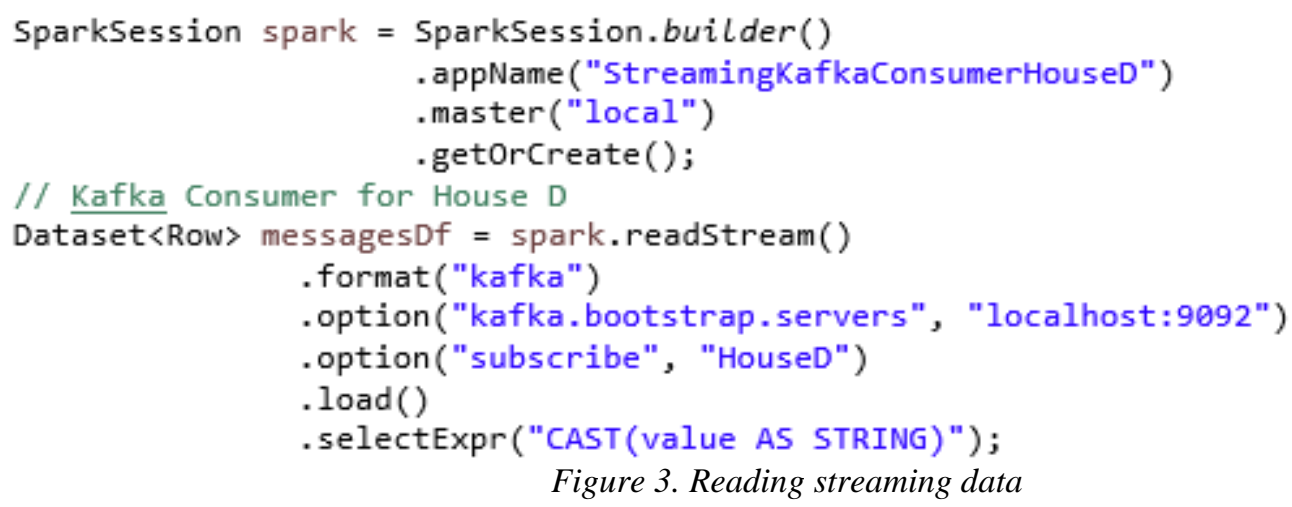

Figure 3. Reading streaming data

\section{Big Data Analytics}

The ultimate goal of collecting large volumes of data is to process and analyze them in order to obtain information and, therefore, value. For this reason, the Big Data Analytics paradigm appeared.

Big data analytics can be applied on sitting data, data that has accumulated during a shorter or longer period of time, on streams of data, or in most cases on a combination of the two. In such a case models are built and validated on accumulated and integrated data from multiple sources and applied in real-time or almost in real-time on streams of data. Algorithms tackle various problems, ranging from sentiment analyses, product recommendations, pattern detections to sophisticated multi-objective problems. The latter are problems that have more than one objective function that needs to be optimized simultaneous. In a big data environment is not uncommon for multi-objective problems to receive constraints and parameters that change with streams of data flowing from different sources at different time resolutions. Algorithms that can address such problems are usually grouped in Machine Learning (ML) packages and tools. Knowledge extraction and incorporation as part of a workflow design is becoming an important component of Big Data analytics as it favors data and algorithm integration. Barba-Gonzalez [4] use both well-known big data products and frameworks including Spark, Kafka, Weka and less-known solutions such as jMetalSP (previously developed by the same authors [5]) for task optimizing or BigML, for Cloud-based Machine Learning processing to demonstrate that annoting big data sources and algorithms can act as a catalyzer for incorporating domain knowledge and improve the analytical process.

The algorithms available to data scientist can be classified in several categories, depending on the data set characteristics as well as the type of problem that needs to be solved, as can be seen in Figure 4.

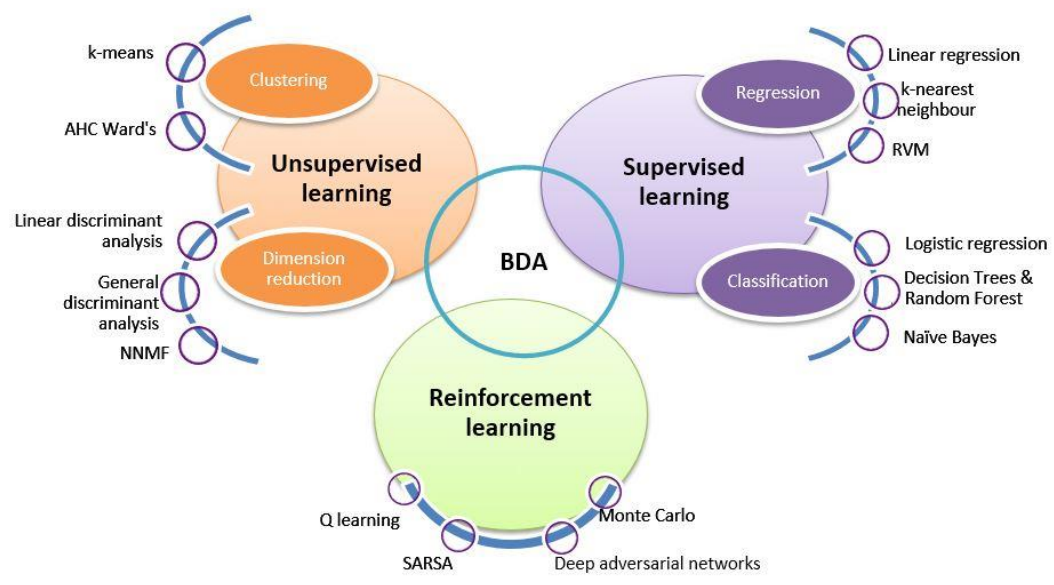

Figure 4. Categories of Big Data Analytics Algorithms 
Some of the more useful and widely used algorithms, including when working with data regarding electricity consumption are the ones for Classification (trying to assign new data points to existing categories), Regression (trying to estimate the relationships between different variables of the data set) and Clustering (identifying similar clusters from the data set). Classification techniques such as Logistic Regression, Support Vector Machines, Naive Bayes or Decision Trees and Random Forests have especially attracted researcher's interest due to their applications' significance and are of particular interest for our research as well. Most of these algorithms can be implemented in both Phyton and PySpark, the latter with which we work for analyzing the data provided by the smart meters.

When working with data streams, classical versions of such techniques, that were designed to work with static datasets, need to be adapted in order to be able to satisfactory deal with "constraints such as bounded memory, single-pass, real-time response, and concept-drift detection" [7]. Several versions of classification or regression algorithms have been proposed such as [8] for Logistical regression, [9] for Naïve Bayes or [10] for Random forest.

To apply a ML algorithm on a stream of data, after we transform the data like in section 3, we define a new dataset with the needed features from messagesDF and train the appropriate algorithm, in this case K-Means (Figure 5):

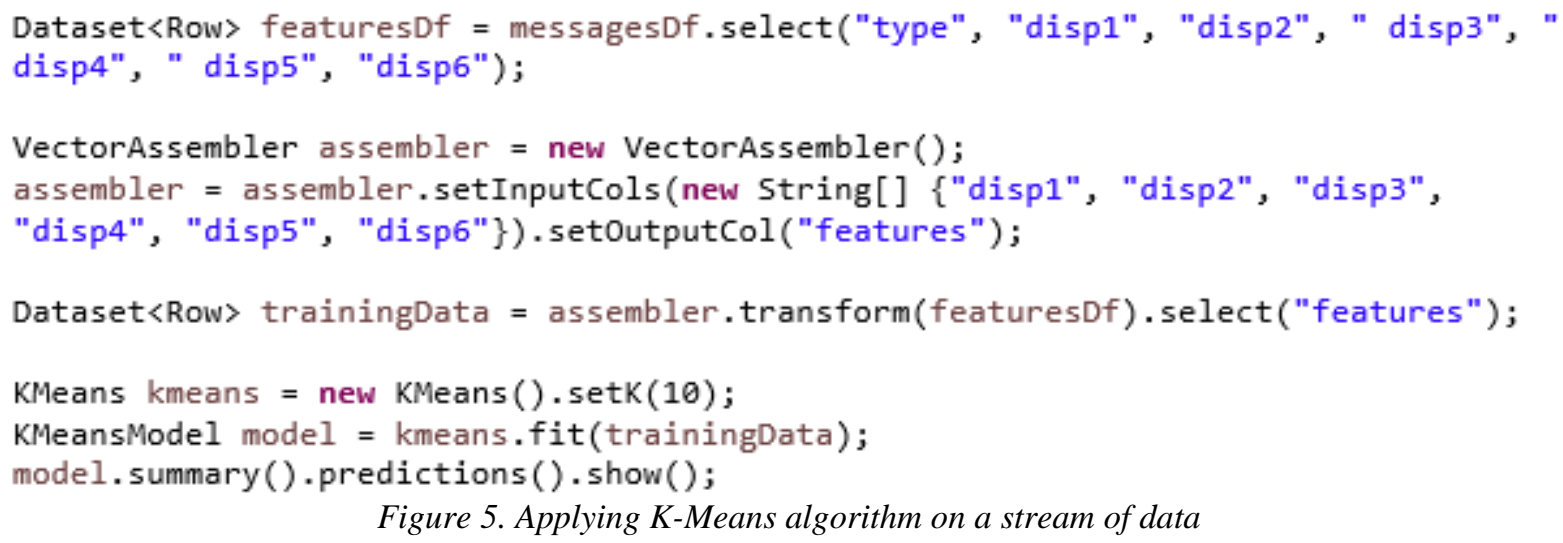

Figure 5. Applying K-Means algorithm on a stream of data

After training the dataset, we need to test the algorithm and then we can start make predictions on data.

\section{Conclusions}

The paper presents the integration architecture of the solution proposed within the SMARTRADE project. We exemplified the use of Spark to process and integrate data streams from a series of smart meters, as well as to implement a clustering algorithm, respectively $\mathrm{K}$ Means.

\section{Acknowledgment}

This paper presents the scientific results of the project "Intelligent system for trading on wholesale electricity market" (SMARTRADE), co-financed by the European Regional Development Fund (ERDF), through the Competitiveness Operational Programme (COP) 2014-2020, priority axis 1 - Research, technological development and innovation (RD\&I) to support economic competitiveness and business development, Action 1.1.4 - Attracting highlevel personnel from abroad in order to enhance the RD capacity, contract ID P_37_418, no. 62/05.09.2016, beneficiary The Bucharest University of Economic Studies. 
www.conferenceie.ase.ro

\section{References}

[1] A. Bara, S.V. Oprea, I. Botha, O.B. Tor., "Conceptual design and architecture of an informatics solution for smart trading on wholesale energy market in Romania", Database Systems Journal. [Online], 2016, vol. VII, no. 4, pp.3-12, Available: http://dbjournal.ro/archive/26/26_1.pdf.

[2] V. Diaconita. "Processing unstructured documents and social media using Big Data techniques”. Economic Research-Ekonomska Istraživanja Journal. [Online], 2015, vol. 28, issue 1, pp.981-993. Available:

https://www.tandfonline.com/doi/pdf/10.1080/1331677X.2015.109511?needAccess=true.

[3] C.Taylor (2018). Structured vs. Unstructured Data. [Online]. Available: https://www.datamation.com/big-data/structured-vs-unstructured-data.html [Accessed March, 2019].

[4] C. Barba-González, J. García-Nieto, M. del Mar Roldán-García, I. Navas-Delgado, A. J. Nebro, and J. F. Aldana-Montes, "BIGOWL: Knowledge centered Big Data analytics," Expert Syst. Appl., vol. 115, pp. 543-556, 2019.

[5] C. Barba-González et al., "jMetalSP: A framework for dynamic multi-objective big data optimization," Appl. Soft Comput., vol. 69, pp. 737-748, Aug. 2018.

[6] P. Ruiz, P. Morales-Alvarez, R. Molina, and A. K. Katsaggelos, "Learning from crowds with variational Gaussian processes," PATTERN Recognit., vol. 88, pp. 298-311, Apr. 2019.

[7] H. Nguyen, Y. Woon, W. Ng. A Survey on Data Stream Clustering and Classification, Knowledge and Information Systems, 2015, vol. 45 no. 3, pp. 535-569 . Available https://www.researchgate.net/publication/269776374_A_Survey_on_Data_Stream_Cluste ring_and_Classification

[8] Z.-W. YinS.-T. HuangG.-R. Xue. Logistic regression for evolving data streams https://www.researchgate.net/publication/290596691_Logistic_regression_for_evolving_ data_streams_classification

[9] M. Bahri, S. Maniu, A. Bifet, A Sketch-Based Naive Bayes Algorithms for Evolving Data Streams, IEEE International Conference on Big Data, Seattle, USA, 2018

[10] H.M Gomes, A. Bifet, J. Read et al, Adaptive random forests for evolving data stream classification, Machine Learning, vol. 106, no. 9-10, pp. 1469-1495, 2018. 